PAPER

\title{
Planning and realisation of complex intentions in patients with Parkinson's disease
}

\author{
M Kliegel, L H Phillips, U Lemke, U A Kopp
}

J Neurol Neurosurg Psychiatry 2005;76:1501-1505. doi: 10.1136/jnnp.2004.051268

See end of article for authors' affiliations

.....................

Correspondence to: Dr Matthias Kliegel, Institute of Psychology, University of Zürich, Schaffhauserstr $15, \mathrm{CH}$ 8006 Zürich; m.kliegel@ psychologie.unizh.ch

Received 4 August 2004 In revised form 14 February 2005 Accepted 24 February 2005

\begin{abstract}
Background: There is some evidence that patients with Parkinson's disease may impaired in prospective memory performance (planning and self initiated realisation of delayed intentions). Little is known about the effect of the disease on distinct phases of prospective memory and the potential mechanisms underlying these effects.

Objective: To investigate intention formation, intention retention, intention initiation, and intention execution of patients with Parkinson's disease and test for the mediating influence of working memory, inhibition, short term retrospective memory, and divided attention.

Methods: 16 patients with Parkinson's disease and 16 age and education matched normal controls were given a complex event based prospective memory task which differentiates four phases of prospective remembering. In addition, participants completed tasks assessing potential cognitive mediators.

Results: On the prospective remembering task, Parkinson patients were impaired in the intention formation phase and showed a trend towards impairment in the intention initiation. In contrast, there were no impairments of retrospective intention retention or the fidelity with which the patients executed their previously developed plan. The group effects were related to interindividual differences in working memory span.

Conclusions: The results suggest that the planning phase of prospective remembering is specifically impaired in Parkinson's disease, and that the impairment is related to working memory deficit. In contrast, even when complex intentions have to be remembered, the retrospective storage of intentions to be performed is not impaired.
\end{abstract}

f someone asked you to specify three memory problems you had last week it is very likely that at least one would concern the delayed execution of an intended action-for example, forgetting to give someone a call. This type of memory has been labelled prospective memory and interest in this rather new field of cognitive psychology is growing.

Kliegel and Martin ${ }^{2}$ have recently summarised three reasons why research on prospective memory is highly relevant: first, prospective memory is theoretically important because it has some properties which make it distinct from the more usual topic of memory research (memory for previous information or episodes, generally known as retrospective memory); second, prospective memory is of great relevance for everyday life; evidence indicates that $50-80 \%$ of everyday memory problems involve prospective memory failures $^{3}$; and third, in line with its importance for independent living, prospective memory is of enormous clinical relevance. Prospective memory has been investigated in a number of clinical conditions, such as Herpes simplex encephalitis, ${ }^{5}$ Korsakoff's syndrome, ${ }^{6}$ early dementia, ${ }^{78}$ schizophrenia, ${ }^{9}$ depression, ${ }^{10}$ and brain injury. ${ }^{11}$ Most of the reported prospective memory deficits have been linked to impaired executive functions such as planning abilities. ${ }^{12}$ Several task analyses have shown that prospective memory involves both the "What did I intend to do?" knowledge of retrospective memory and the planning abilities of executive functions..$^{13} 14$

Most studies of memory performance in patients with Parkinson's disease have so far focused exclusively on retrospective memory. ${ }^{15}{ }^{16}$ While relatively simple short term retrospective memory storage requirements do not seem to be impaired in Parkinson's patients, ${ }^{17}$ some executive functions have been reported to be disturbed, especially planning. ${ }^{13} 18$
However, despite these theoretical reasons to predict prospective memory impairments in affected patients, there is only one study which systematically investigates the effects of Parkinson's disease on prospective memory.

Katai et $a l^{19}$ investigated differential performance of Parkinson patients on event based and time based prospective memory tasks. In the event based task, the participant was asked to perform an action whenever a target word was presented. In the time based task, the participant was asked to perform the same action at a specified time. Patients with Parkinson's disease were impaired in the event based prospective memory task, but were not impaired in the time based task. Analysing memory for the instructions, Katai et al found that the impairment in event based prospective memory was not the result of forgetting the content of the instructions, but a failure to retrieve the intention spontaneously when the target appeared. Accordingly, the retrospective component of prospective memory seemed to be preserved whereas the prospective component was impaired in Parkinson patients. However, the investigators discuss the relatively simple retrospective component of their task as an explanation for the absence of impairment on the retrospective component of the prospective memory task. Katai et al speculate that deficits in working memory resources in the Parkinson patients could have been responsible for their findings. ${ }^{19}{ }^{20}$ Concerning the time based prospective memory task, Katai et al proposed that the administration of levodopa in their group might have compensated for the usually observed underestimation of the length of time intervals in Parkinson's disease. ${ }^{19} 21$ Moreover, having only two time based responses might have led to a ceiling effect which made it impossible to detect group differences. 
It was our aim in the present study to continue and extend this line of research. Therefore, Parkinson's patients and control participants were compared in their performance on a more complex event based prospective memory task that increases demands on retrospective memory and allows us to disentangle four phases of prospective remembering-intention formation, retrospective intention retention, appropriate intention initiation, and intention execution..$^{22}$ Thus a more fine grained analysis of potential deficits becomes possible. In addition, we also aimed to address Katai's speculation ${ }^{19}$ on the role of working memory resources by directly assessing working memory capacity (as well as attention, short term memory, and inhibition). By covarying these potential factors of influence we tested the hypothesis that working memory deficits might be responsible for potential group differences between Parkinson patients and controls in the prospective memory process.

\section{METHODS \\ Participants}

Participants were 16 patients with Parkinson's disease (PDs; five female, 11 male; age range 49 to 69 years (mean (SD), 61.1 (6.9) years); disease duration 0.5 to 11 years (4.81 (3.00) years), and 16 matched healthy controls (HCs; five female, 11 male; age range 50 to 74 years (62.6 (9.1) years). All were right handed and none had a history of psychiatric or cardiovascular disease or drug or alcohol abuse. None of the HCs had a history of neurological disease. The PDs were Hoehn and Yahr stage $1(n=10)$ or $2(n=6)$ when on drug treatment. Fifteen patients were treated with a combination of L-dopa and pergolide (a dopamine D1 and D2 receptor agonist) and one patient was taking pergolide only. The mean (SD) dose of L-dopa was 417.33 (145.43) mg/day (range 185 to 700 ), and of pergolide, 4.75 (1.70) $\mathrm{mg} /$ day (range 2 to 8 ). Two patients for whom a "wearing off" phenomenon had been reported were also being treated with entacapone (a COMT inhibitor; patient 1, $800 \mathrm{mg} / \mathrm{d}$; patient 2, $500 \mathrm{mg} / \mathrm{d}$ ). No patient was taking any anticholinergic drugs. No patients showed an "on-off" phenomenon. We included no patients with depression (inclusion criterion, Beck depression inventory $(\mathrm{BDI})<11$ ) and no patients with dementia (inclusion criterion, mini-mental state examination (MMSE) score $>24$ ).

The PDs were taken off all antiparkinsonian drugs for at least 12 hours before testing*. All participants gave their informed consent before taking part in the study. The PD and HC groups did not differ with respect to their education (PDs: 11.0 (2.4) years $v$ HCs: $11.4(1.7)$ years; $F(1,29)=0.36$, NS), nor their premorbid intelligence level as measured by a verbal intelligence test (MWT-B ${ }^{25}$ : PDs: 113.3 (13.5) v HCs: 118.6 $(13.8) ; \mathrm{F}(1,30)=1.22$, NS).

\section{Procedure and materials}

\section{Overview of the prospective memory procedure}

The complex prospective memory task ${ }^{11} 2226$ was assessed in four phases. In the first phase, the participants were instructed and required to develop an explicit intention (intention formation: What is the participant planning to do?). In the second phase, after some distractor tasks retrospective memory for the previously developed intention was tested (intention retention: Does the participant still know the content of

* To control for medication effects a 12 hour washout phase for antiparkinsonian drugs was chosen. Although this is in line with current standards and comparable studies ${ }^{23} 24$ a longstanding influence of drug treatment cannot be completely ruled out. However, given the increase of motor symptoms associated with an ongoing off-medication phase, longer withdrawal times might not have been tolerable for patients and it was assumed they would substantially reduce the patients' compliance. his/her intention?). In the third phase (after some distractor tasks) the plan had to be self initiated (intention initiation: Does the participant remember to initiate task execution at the appropriate moment?). In the fourth phase, the previously planned intention had to be executed on the participant's initiative (intention execution: (a) intention fidelity: Does the participant actually follow his/her previously stated intention? and (b) self initiated actions: Does the participant remember to carry out the intended actions?).

\section{Detailed description of prospective memory procedure Intention formation phase}

Following Kliegel et $a l_{,}^{22}$ at the beginning participants were instructed that, at a certain point in this session, they would have to fill out a personal information questionnaire (as noted below, this was the cue for initiating the complex prospective memory task set). The participants were informed that this would take place later in the session.

\section{The subtasks}

The complex prospective memory paradigm and the rules were then explained to the participants. They were asked to carry out, on their own, six subtasks in a period of six minutes. The six subtasks were divided into two similar sets (sets A and B) of three (finding words, solving arithmetic problems, and writing down the names of pictures). Each subtask was designed so that it would need more than a minute to complete (see Kliegel et al ${ }^{1427}$ for more details).

\section{The rules}

The rules indicated that all six subtasks were to be initiated within the given time limit of six minutes. Thus the rules were designed to require and reward five self initiated switches from working on one type of task to another type of task within the available six minute period. One major rule to obey was that the participants were not allowed to do two subtasks (A) and (B) of the same type (words, maths, or pictures), one after the other.

\section{Test of instruction comprehension}

Afterwards, the participants were tested on the recall of the instructions and the experimenter continued to review the task demands until the participants were fully aware of the rules and could recall them perfectly (which all participants were able to do also at the end of the experiment).

\section{Prospective memory instruction}

The participants were then told that they would have to remember to start this task set by themselves after answering the question about their date of birth in the "participant information form" that had been explained previously. In addition, they were told that they would have to remember on their own to follow their plan and to remember to switch to all six subtasks.

\section{Explicit intention formation}

Next, the participants were asked to develop an explicit intention of how they intended to perform this complex multiple task set. The intention formation had to be verbal and was recorded on a tape recorder. In accordance with Kliegel et $a l,{ }^{22}$ the elaborateness of the intention was analysed in terms of a score that included three main features: (1) the number of rules included in the participant's intention; (2) whether the participant specified the sequence of subtasks; and (3) whether the participant included explicit specification of the 
Table 1 Differences in divided attention, short term memory, working memory, and inhibition between Parkinson's patients and controls

\begin{tabular}{lllll}
\hline Variable & $\begin{array}{l}\text { Patients } \\
(\mathbf{n}=16)\end{array}$ & $\begin{array}{l}\text { Controls } \\
(\mathbf{n}=16)\end{array}$ & $\boldsymbol{F}$ Value & $\mathbf{n}^{\mathbf{2}}$ \\
\hline Divided attention & $787.4(149.9)$ & $696.5(113.6)$ & $3.65^{+}$ & 0.11 \\
Short term memory & $7.3(1.8)$ & $7.3(2.1)$ & 0.00 & 0.00 \\
Inhibition & $52.6(31.9)$ & $31.1(9.2)$ & $6.72^{*}$ & 0.18 \\
Working memory & $13.8(9.3)$ & $23.94(13.1)$ & $5.86^{*}$ & 0.17 \\
\hline & & & & \\
Values are mean (SD). \\
$+\mathrm{p}=0.06,{ }^{*} \mathrm{p}<0.05$.
\end{tabular}

amount of time to be spent on each step (see Kliegel et al ${ }^{22}$ for more detail).

\section{Intention retention phase \\ Distractor activities}

The following intention retention phase lasted about 20 minutes and was filled with distractor activities (that is, cognitive tests described below that will be used as covariates).

\section{Retrospective memory for the intention}

Afterwards, the participants had to recall their intention for the complex prospective memory task set (intention retention), which was scored for accuracy relative to the plan previously stated in the intention formation phase.

\section{Intention initiation phase \\ Distractor activities}

This was followed by another delay of approximately 20 minutes filled with distractor activities (see below). In the middle of this delay, there was a five minute break.

\section{Initiation}

The participants were then given the participant information questionnaire. After having answered the question about their date of birth, participants were supposed to initiate their intention concerning the performance of the complex prospective memory task set by themselves (intention initiation). The score was based on whether or not the participants initiated the tasks after having written their date of birth on the participant information form $(0=$ not initiated; $\mathrm{l}=$ initiated).

\section{Intention execution phase}

If the participants did not start the tasks after having finished the entire questionnaire, the experimenter prompted them to do so and asked if they could recall when they were supposed to have started the six tasks (which all participants were able to do).

\begin{tabular}{lll} 
Table 2 & Predictors of group effects in intention formation \\
\hline & \multicolumn{2}{l}{ Intention formation } \\
\cline { 2 - 3 } Group effect in: & $\boldsymbol{F}$ Value & $\eta^{2}$ \\
\hline Original ANOVA & $12.16^{* *}$ & 0.29 \\
Group effect when covarying ... & $7.01^{*}$ & 0.20 \\
$\quad$ Divided attention & $17.35^{* * *}$ & 0.37 \\
Short term memory & $8.41^{* *}$ & 0.23 \\
Inhibition & $4.33^{+}$ & 0.14 \\
Working memory & & \\
\hline${ }^{+} p<0.10,{ }^{*} p<0.05,{ }^{* *} p<0.01,{ }^{* * *} p<0.001$. \\
ANOVA, analysis of variance.
\end{tabular}

Finally, intention execution was measured by two scores, with the first one, intention fidelity, indicating how well participants put their previously formed intention into action. The second measure, self initiated switching, indicating overall task performance, was derived by subtracting the number of breaks of the second rule ("You are not allowed to do two subtasks (A) and (B) of the same type one after the other") from the number of subtasks that were started (of six possible tasks).

\section{End of procedure}

After having worked on the complex task for six minutes, the participants filled out the remainder of their participant information questionnaire and were debriefed.

\section{Cognitive resources (distractor activities)}

Divided attention was measured with a computerised standardised attention task (TAP, test battery of attention ${ }^{28}$ ). The divided attention task measures the ability to divide attention between two sensory modes (dual task performance), here visual and auditory stimulation. The visual task was to detect a square pattern, consisting of four of eight crosses presented on the computer screen. In the auditory task, two tones alternated, and the participant had to detect irregularities in this two tone sequence. The dependent variable was reaction times for correct responses, with higher reaction times indicating worse divided attention.

To assess short term memory span, we administered the digit span forward subscale from the Wechsler adult intelligence scale (WAIS- $\mathrm{R}^{29}$ ). Here, several rows of digits of increasing span size (three to nine digits) were presented orally and participants' task was to reproduce the sequence of digits in the correct order. Short term memory span was the number of correctly recalled sequences, with higher scores indicating better short term memory.

To determine working memory capacity we applied the operation span measure developed by Turner and Engle. ${ }^{30}$ In this task participants were asked to read and verify a simple maths problem (such as $(4 / 2)-1=1$ ) and then read a word after the operation (such as SNOW). After a series of problems and words had been presented, the participants were asked to recall the words that followed each operation in correct order. The number of operation-word strings in a sequence was increased and decreased randomly (span sizes two to six) to measure the participant's operation span. The dependent variable was the number of correctly recalled words in correct span trials, with higher scores indicating greater working memory capacity.

Finally, the Stroop task was used to measure inhibition. ${ }^{31}$ The baseline trials consisted of colour bars (red, blue, green, and yellow), with participants asked to name the colour as quickly as possible. The interference trials consisted of the corresponding colour name words printed in mismatched colours, and again participants were asked to name the colour of the stimuli as quickly as possible. The dependent variable was the time difference between the interference condition and baseline. Higher scores indicate poorer inhibition.

\section{RESULTS}

\section{Cognitive resources}

Descriptive statistics can be seen in table 1. The ANOVAs revealed significant deficits in the Parkinson patients in working memory capacity and inhibition. The group effect on divided attention approached significance. No group difference was obtained in the short term memory span measure. 


\section{Complex prospective memory performance}

Using one way analysis of variance (ANOVA) we then compared Parkinson patients and controls with regard to performance in each distinct phase of the complex prospective memory paradigm. Where these ANOVAs revealed significant group differences we investigated the influence of potential underlying cognitive mechanisms by covarying divided attention, short term memory, inhibition, and working memory. $†$

\section{Intention formation}

As revealed by an initial ANOVA, there was a significant effect of group on intention formation indicating that the control group developed more complex intentions than the patient group (mean (SD): $\mathrm{M}_{\mathrm{PD}}=7.8$ (2.2) $v \mathrm{M}_{\mathrm{HC}}=13.3$ (5.8); for the $F$ values and effect sizes see table 2 ). The following ANCOVAs showed that covarying divided attention as well as inhibition reduced the effect by $31 \%$ (divided attention) or $22 \%$ (inhibition), respectively. In contrast, covarying short term memory even increased the magnitude of the group effect $(\mathrm{F}=12.16$ in the original ANOVA to $\mathrm{F}=17.35$; that is, a $30 \%$ increase in $\eta^{2}$ ). However, covarying working memory span strongly reduced the highly significant group effect on the intention formation measure to almost non-significance $(\mathrm{F}=4.33, \mathrm{p}=0.05)$. Considering the decrease in $\eta^{2}$, working memory explained $52 \%$ of the group related variance in complex prospective memory intention formation.

\section{Intention retention}

With regard to plan retention, both groups showed a very high and comparable level of retrospective memory of their planned intention: $\mathrm{M}_{\mathrm{PD}}=92.19 \quad(13.86) \% \quad v \quad \mathrm{M}_{\mathrm{HC}}=88.46$ $(21.93) \%, F<1$.

\section{Intention initiation}

Of those in the control group, $63 \%$ initiated the intention at the appropriate moment, compared with $31 \%$ of the Parkinson group. Non-parametric analysis showed that this difference approached significance $\left(\chi^{2}(1)=3.19, p=0.07\right)$.

\section{Intention execution: intention fidelity}

The ANOVA on the intention fidelity scores showed a relatively low plan fidelity and no difference between the groups: $\mathrm{M}_{\mathrm{PD}}=58.33(26.53) \% v \mathrm{M}_{\mathrm{HC}}=59.14(31.61) \%, \mathrm{~F}<\mathrm{l}$.

\section{Intention execution: self initiated switching}

Regarding the switching score, the group difference in means was not statistically significant: Controls performed on average 4.00 (1.10) subtasks and Parkinson patients performed on average 3.38 (1.41) subtasks $(F(1,31)=1.96$, $\mathrm{p}=0.17$ ).

\section{DISCUSSION}

The results of this study show that patients with Parkinson's disease were particularly impaired in the intention formation phase of the prospective memory process. There was also a trend towards group differences in the intention initiation component. In contrast, there were no impairments in retrospective intention retention, the fidelity with which the patients executed their previously developed plan or the self initiated switching component.

†We decided to apply analysis of covariance (ANCOVA) in order to address directly the issue of potential mediators for obtained group differences in the ANOVAs. Using simple correlation analyses, partial correlations, or multiple regression analyses, respectively, as done in previous work ${ }^{22}$ did not change the results.
The latter seems to be in contrast to theories of Parkinson's disease that highlight deficits of internal control and timing ${ }^{32}$ but in line with the first study on prospective memory in Parkinson's disease by Katai et al, ${ }^{19}$ who found no group difference in a time based task which also required task switching. However, Katai et al acknowledge potential limitations of their findings by possible compensatory effects of continuing Parkinson medication and potential ceiling effects of their time based paradigm. Similarly, our nonsignificant switching result may also be limited by long term medication effects, as the descriptive results seem to indicate a small effect in favour of the control participants but see footnote *. The current findings of no significant effect on switching is at least evidence against a strong deficit of internal control and timing in Parkinson's disease. Clearly, more research is needed to address these findings in the context of prospective memory.

The trend towards poorer intention initiation appears to be in line with Katai et al, who found an effect of Parkinson's disease on self initiation of an event based task. Extending the literature, the present results suggest that the major source of impairment may be in poorer formulation of detailed intentions. In line with the findings of Katai et al, the present data robustly underline the fact that these trends were not attributable to impaired retrospective memory for the intention. In Katai's study the lack of group differences in retrospective memory might be a result of ceiling effects. In the present study, preserved retrospective memory for the intention was also found for a very detailed plan that participants had to recall retrospectively.

Moreover as the plan fidelity score indicates, Parkinson patients were not impaired in the ability to follow their original plan. However, as indicated by the intention formation score, this plan was significantly worse compared with the controls' plans. Thus our findings support the conclusion of a specific prospective memory deficit in Parkinson's patients in conjunction with a preserved retrospective memory component-even for relatively complex intentions. In addition, the results specify the locus of this deficit by revealing a reliable impairment in the intention formation phase of complex prospective remembering. Considering previous studies on planning performance in Parkinson's disease this finding is consistent with most of the literature. Among others, Owen et $a^{33}{ }^{34}$ have pointed out that "parkinsonian patients are impaired on tasks that involve self-directed behavioral planning" (p 127).

A second novel aspect of the present results concerns the role of cognitive resources potentially underlying the obtained group effects. Here, working memory capacity in particular was found to play an important role. With regard to the group effect in intention formation, covarying working memory capacity reduced the group effect by $50 \%$, resulting in a non-significant effect of Parkinson's disease. This supports the conclusion that Parkinson related interindividual differences in working memory capacity at least partly drive impairments in prospective memory planning. This is in line with reports on the role of working memory in planning deficits in Parkinson patients. ${ }^{32}$

\section{Conclusions}

In sum, the present findings support recent reports that the self directed formation of delayed intentions may be impaired in Parkinson's disease. Considering the relevance of this cognitive process for independent living, ${ }^{35}$ the results underline the need for further investigation of the components that seem to be of particular difficulty for Parkinson patients, as well as of potential mechanisms that may underlie these effects. Our study indicates that working memory is an important factor underlying such deficits in Parkinson's disease. 


\section{Authors' affiliations}

M Kliegel, U Lemke, Department of Gerontopsychology, University of Zurich, Zurich, Switzerland

L H Phillips, School of Psychology, University of Aberdeen, Aberdeen, UK

Ute A Kopp, Department of Neurology, University Hospital Charité, Berlin, Germany

Competing interests: none declared

\section{REFERENCES}

1 Ellis J, Kvavilashvili L. Prospective memory in 2000: past, present, and future directions. Appl Cogn Psychol, 2000;14:S1-9.

2 Kliegel M. Martin M. Prospective memory research: why is it relevant? Int J Psychol 2003:38:193-4.

3 Crovitz HF, Daniel WF. Measurements of everyday memory: toward the prevention of forgetting. Bull Psychonom Soc 1984;22:413-14.

4 Terry WS. Everyday forgetting: data from a diary study. Psychol Rep 1988:62:299-303.

5 Sgaramella TM, Borgo F, Fenzo F, et al. Memory for and execution of future intentions: evidence from patients with Herpes simplex encephalitis. Brain Cogn 2000;43:388-92.

6 Brunfaut E, Vanoverberghe $V, d^{\prime} Y$ dewalle $G$. Prospective remembering of Korsakoffs and alcoholics as a function of the prospective-memory and ongoing tasks. Neuropsychologia 2000;38:975-84.

7 Huppert FA, Beardsall L. Prospective memory impairment as an early indicato of dementia. J Clin Exp Neuropsychol 1993;15:805-21.

8 Huppert FA, Johnson T, Nickson J. High prevalence of prospective memory impairment in the elderly and in early-stage dementia: findings from a population-based study. Appl Cogn Psychol 2000;14:S63-81.

9 Meissner F, Hacker W, Heilemann H. Gedachtnisleistungen und Instruktionseffekte bei Schizophrenie: eine vergleichende Untersuchung an chronisch Schizophrenen und Gesunden. [Memory performance and instruction effects in schizophrenia: a comparative study of chronic schizophrenic patients and healthy controls], Psychiatr Prax 2001;28:180-8.

10 Rude SS, Hertel PT, Jarrold W, et al. Depression-related impairments in prospective memory. Cogn Emotion 1999;13:267-76.

11 Kliegel M, Eschen A, Thone-Otto AIT. Planning and realization of complex intentions in traumatic brain injury and normal aging. Brain Cogn 2004;56:43-54.

12 Smith EE, Jonides J. Storage and executive processes in the frontal lobes. Science 1999:283:1657-61.

13 Ellis J. Prospective memory or the realization of delayed intentions: a conceptual framework for research. In: Brandimonte M, Einstein GO, McDaniel MA, eds. Prospective memory: theory and applications. Mahwah: Lawrence Erlbaum, 1996: 1-22.

14 Kliegel M, Martin M, McDaniel MA, et al. Complex prospective memory and executive control of working memory: a process model. Psychol Beitrage 2002;44:303-18.
15 Breen EK. Recall and recognition memory in Parkinson's disease. Cortex 1993;29:91-102.

16 Dubois B, Pillon B. Cognitive deficits in Parkinson's disease. J Neurol 1997;244:2-8.

17 Boller F, Muggia S. Non-Alzheimer dementias. In: Denes G, Pizzamiglio L, eds. Handbook of clinical and experimental neuropsychology. Hove: Psychology Press, 1999:747-74.

18 Morris RG, Downes JJ, Evenden JL, et al. Planning and spatial working memory in Parkinson's disease. J Neurol Neurosurg Psychiatry 1988;51:757-66

19 Katai S, Maruyama T, Hashimoto T, et al. Event based and time based prospective memory in Parkinson's disease. J Neurol Neurosurg Psychiatry 2003;74:704-9.

20 Bradley VA, Welch JL, Dick DJ. Visuospatial working memory in Parkinson's disease. J Neurol Neurosurg Psychiatry 1989;52:1228-35.

21 Pastor MA, Artieda J, Jahanshahi $M$, et al. Time estimation and reproduction is abnormal in Parkinson's disease. Brain 1992;115:211-25.

22 Kliegel M, McDaniel MA, Einstein GO. Plan formation, retention, and execution in prospective memory: A new approach and age-related effects. Mem Cogn 2000;28:1041-9.

23 Defer GL, Widner H, Marie RM, et al. Core assessment program for surgical interventional therapies in Parkinson's disease (CAPSIT-PD). Move Disord 1999; 14:572-84

24 Fern-Pollak L, Whone AL, Brooks DJ, et al. Cognitive and motor effects of dopaminergic medication withdrawal in Parkinson's disease. Neuropsychologia 2004;42:1917-26.

25 Lehrl S. Der Mehrfachwahlwortschatztest MWT-B. Erlangen: Straube, 1977

26 Shallice T, Burgess PW. Deficits in strategy application disorder following frontal lobe damage in man. Brain 1991;114:727-41.

27 Kliegel M, Martin M, Moor C. Prospective memory and aging: is task importance relevant? Int J Psychol 2003;38:207-14.

28 Zimmermann P, Fimm B. Testbatterie zur Aufmerksamkeitsprufung (TAP). Version 1.02c. Herzogenrath: Psytest, 1994.

29 Wechsler D. Wechsler adult intelligence scale - revised (WAIS-R). New York: Psychological Corporation, 1981.

30 Turner ML, Engle RW. Is working memory capacity task dependent? J Mem Lang 1989;28:127-54.

31 Houx PJ, Jolles J, Vreeling FW. Stroop interference: aging effects assessed with the Stroop color-word test. Exp Aging Res 1993; 19:209-24.

32 Jahanshahi $M$, Frith $C D$. Willed action and its impairments. Cogn Neuropsychol 1998;15:483-533.

33 Owen AM, James M, Leigh PN, et al. Fronto-striatal cognitive deficits at different stages of Parkinson's disease. Brain 1992;115:1727-51.

34 Owen AM, Sahakian BJ, Hodges JR, et al. Dopamine-dependent frontostriatal planning deficits in early Parkinson's disease. Neuropsychology 1995;9:126-40.

35 Phillips LH, MacLeod MS, Kliegel M. Adult aging and cognitive planning. In: Morris R, Ward G, eds. The cognitive psychology of planning. Hove: Psychology Press, 2005:111-34. 